\title{
La Producción y el Comercio Internacional de Cemento en el Mundo $y$ en España
}

\section{Producción}

El panorama económico mundial en el período 1960-61 no era ciertamente esperanzador; se anunciaba la crisis del dólar, de la que se vuelve a hablar ahora; existían importantes huelgas en Estados Unidos, sobre todo en la industria del acero; los problemas de la incompatibilidad del Mercado Común y la Zona de Libre Comercio en Europa quedaron sin resolver, y sólo, a fines del último año, se inician conversaciones en virtud de las cuales se intenta que Inglaterra, y con ella los demás países «periféricos», fortalezcan sus lazos de unión con la Europa de los «seis», consecuencia, en gran parte del «clima» de incomprensión y tensión internacional entre el «bloque oriental» y el «occidental».

Desde el punto de vista positivo, es importante la incorporación de los Estados Unidos y Canadá a las instituciones europeas efectuadas en 1961, sobre todo, a través de la extinguida O. E. C. E. y, consiguiente constitución del nuevo organismo O. C. D. E., que se planteará, como un conjunto, una serie de importantes problemas como la disociación y desajuste entre las políticas-económica sobre todo-de Norteamérica y Europa: también entre los «seis» de la C. E. E. y «los siete» de la E. F. T. A. y, sobre todo, el crucial de la ayuda a los países subdesarrollados o en vías de desarrollo, que agrupan no menos de 1.700 millones de personas, de un total mundial de 2.800 millones.

Esta situación y el problema de fortalecimiento de las nacionalidades (Iberoamérica) y creación de otras nuevas e independientes (Africa negra), impregnan de inquietud política el ambiente universal, necesitado de reajustar su actividad a las nuevas circunstancias, haciendo compatible su propio bienestar interior con la necesidad de cada «bloque», oriental u occidental, de incorporar estos nuevos países a su esfera de influencia.

Y sin embargo, y a pesar de que el período 1960-61 ha venido caracterizado por la situación política mencionada de espectativa, reajuste e inquietud, el ritmo de las producciones mundiales no ha disminuído respecto a años anteriores, según el último estudio de las Naciones Unidas, que estima que 1960 ha sido un año de prosperidad para Europa occidental, caracterizado por:

Gran expansión de la demanda de productos de exportación y de la demanda interior.

Gran elasticidad de la oferta.

Estabilidad relativa de los precios; y

Situación favorable en la mayor parte de los países, en cuanto a reserva de divisas.

En cifras se llega a establecer que en Europa occidental:

a) La producción total aumentó en un 6,5\% en 1960 respecto a 1959, en cuyo año el incremento fue de un $4 \%$ sobre el anterior. 
Es de indicar que en el sector de la vivienda se observa una contracción en la mayoría de los países considerados, consecuencia de la política económica seguida, que pretende frenar la demanda excesiva, o por razón de escasez de materiales, de mano de obra o de ambas.

b) La producción industrial aumentó en un $11 \%$ entre 1959 y 1960, porcentaje alto respecto al $7 \%$-no llegó-entre 1958 y 1959.

c) En la industria del cemento, y según los estudios del Cembureau (The Cement Statistical And Technical Association), en el año 1959 la producción total mundial alcanzó los 290 millones de t, pero en 1960 se ha elevado a 315 millones, lo que supone un aumento del 11,5\%, porcentaje, por tanto, mayor al del índice general de la producción industrial europea.

No hay que olvidar que los aumentos en producción fueron de un $12 \%$ en el año 1959 respecto a 1958, y de un $5 \%$ de 1958 respecto a 1957. Por lo tanto, se ha conseguido en 1960-se desconocen aún las cifras de 1961-un aumento muy significativo, pero superior, desde luego, a las tasas que se consideran como muy favorables para este tipo de industria pesada.

Parece interesante recoger aquí la distribución de los aumentos de producción experimentados en el mundo, sistematizados por orden decreciente, en cuanto a la tasa de crecimiento en la producción, en la siguiente forma:

\section{Asia y Oceanía.}

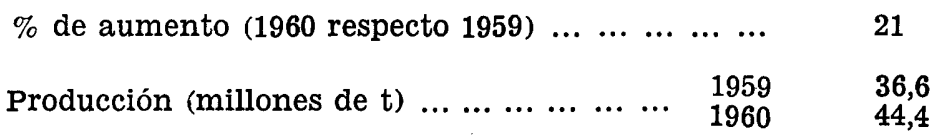

Hay que indicar que el mayor incremento corresponde a Japón, con un $31 \%$ del total del grupo y un aumento en valores absolutos de 5,3 millones de toneladas, debiendo mencionarse también a la India y el Irán. También es importantísimo el avance experimentado por el bloque comunista, considerando como tal a los países asiáticos y europeos de esta tendencia, que pasó en el período considerado de 73,4 millones de toneladas, a 86,6, lo que supone un incremento del $18 \%$, es decir, casi el doble del promedio mundial, siendo de considerar por su importancia los siguie.ntes países:

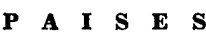

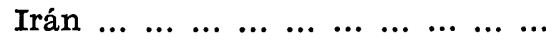

Japón $\ldots \ldots \ldots \ldots \ldots \ldots$

China .......................

Alemania Oriental del Este ........

Polonia $\ldots \ldots \ldots \ldots \ldots \ldots$

Corea del Norte.............

India $\ldots . . . \ldots \ldots \ldots$

Rusia...........

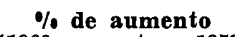

$(1960$ respecto a 1959$)$

\section{$50 \%$}

$31 \%$

$26 \%$

$26 \%$

$24 \%$

$22 \%$

$13 \%$

$17 \%$
Producción
(millones de $t$ )

1960

$\overline{22,4}$

15,5

6,6

7,6

45,5

\section{Europa.}

Las cifras son como sigue:

\begin{tabular}{|c|c|}
\hline Producción (millones de $\mathrm{t}$ ) & $\begin{array}{c}1959 \\
1960\end{array}$ \\
\hline
\end{tabular}

Los países correspondientes al Cembureau suponen un porcentaje muy fuerte en la producción total de cemento europeo, ya que en 1959 totalizaron 88 millones de toneladas, y 93,4 en 1960 , frente a las cifras totales indicadas más arriba. 
La distribución por países de estos incrementos es como sigue:

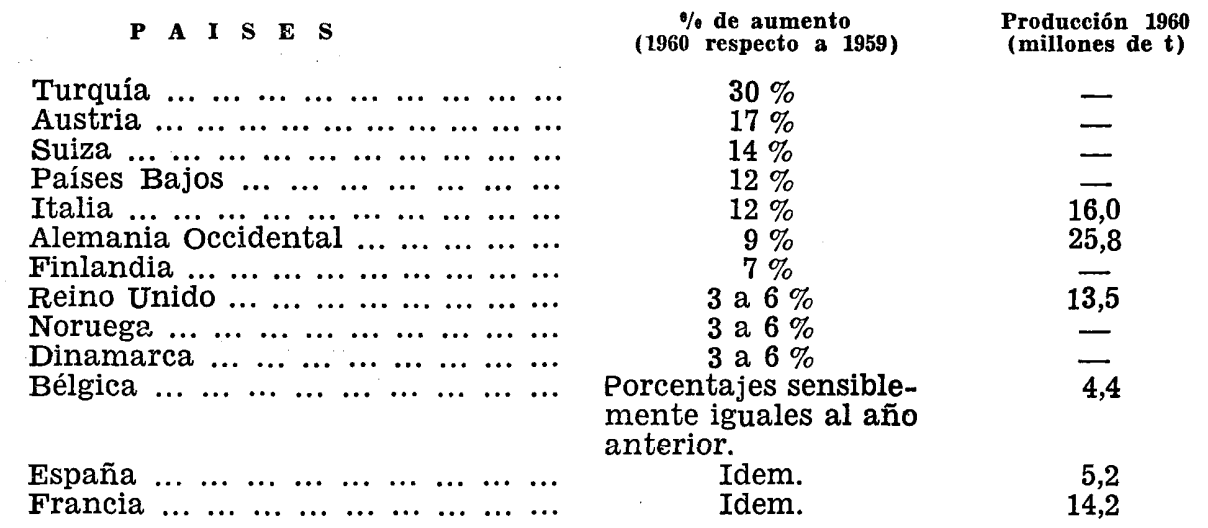

En cuanto a España, las cifras de producción en los últimos años han sido como sigue:

España.

$\begin{array}{lllllll}\text { \% de aumento (1960 respecto } 1959) & \ldots & \ldots & \ldots & \ldots & & \mathbf{0 , 5} \\ & & & \ldots & 1959 & \mathbf{5 , 2} \\ \text { Cantidad producida (millones de t) } & \ldots & \ldots & \ldots & 1960 & \mathbf{5 , 2}\end{array}$

\begin{tabular}{|c|c|c|c|c|c|c|c|}
\hline \multicolumn{5}{|c|}{ 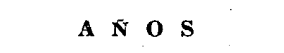 } & \multicolumn{2}{|c|}{ P ROD U C CIO N } & $\begin{array}{c}\% \text { de aumento } \\
\text { (cada año respecto al anterior) }\end{array}$ \\
\hline 1950 & $\ldots$ & $\ldots$ & $\ldots$ & $\cdots$ & 2,103 & 100 & - \\
\hline 1951 & $\ldots$ & $\ldots$ & $\ldots$ & $\ldots$ & 2,323 & 110 & 9 \\
\hline 1952 & $\ldots$ & $\ldots$ & $\ldots$ & $\ldots$ & 2,457 & 117 & 5 \\
\hline 1953 & $\ldots$ & ... & $\ldots$ & ... & 2,765 & 131 & 11 \\
\hline 1954 & $\ldots$ & ... & $\ldots$ & ... & 3,323 & 159 & 16 \\
\hline 1955 & $\ldots$ & $\ldots$ & $\ldots$ & $\ldots$ & 3,752 & 178 & 11 \\
\hline 1956 & $\ldots$ & $\ldots$ & $\ldots$ & ... & 3,975 & 189 & 5 \\
\hline 1957 & $\ldots$ & $\ldots$ & $\ldots$ & ... & 4,487 & 213 & 11 \\
\hline 1958 & $\ldots$ & $\ldots$ & $\ldots$ & ... & 4,817 & 229 & 6 \\
\hline 1959 & $\ldots$ & $\ldots$ & $\ldots$ & $\ldots$ & 5,221 & 248 & 7 \\
\hline 1960 & $\ldots$ & $\ldots$ & $\ldots$ & $\ldots$ & 5,247 & 249 & 0,5 \\
\hline
\end{tabular}

Es de indicar que el reducido aumento del 0,5\% experimentado en el período 1959-60 se debe, sobre todo, a la entrada en acción de las medidas del Plan de Estabilización, que produjo una contracción en las ventas en el primer semestre de 1960 de un $16 \%$, iniciándose la recuperación en el segundo semestre con un aumento en la capacidad de producción, en 1960, de 552.000 toneladas (413.000 de ampliaciones y 139.000 de nuevas fábricas), siendo, además, las previsiones para 1961 optimistas, pues la producción llegará posiblemente a los $5.400 .000 \mathrm{t}$, como consecuencia del esfuerzo que realiza la industria y las nuevas inversiones que han totalizado, en 1960, 895 millones de pesetas.

\section{Africa.}

No existe variación sensible en la producción africana de cemento, que también en 1958 totalizó algo más de nueve millones $(9.088 .000)$ de toneladas.

América.

$$
\begin{aligned}
& \text { \% de variación (disminución } 1960 \text { respecto 1959). } 3 \\
& \text { Producción (millones de t)... ... ........... } \begin{array}{lllll}
1959 & 7960 & 79,1 \\
76,8
\end{array}
\end{aligned}
$$

La baja sufrida en la producción del Continente americano como conjunto, se debe, sobre todo, a la menor producción de Estados Unidos, que pasó de 56,8 millones de t, en 1959, a 53,5 millones, en 1960, con una disminución porcentual del $6 \%$. 
En cuanto a la clasificación de países, es como sigue:

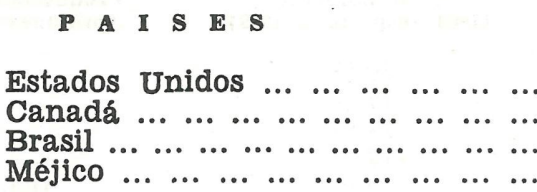

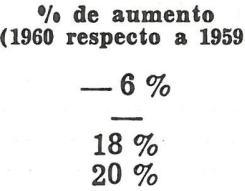

Producción 1960 (millones de $t$ )

53,5

15,3

$\begin{array}{r}15,3 \\ 4,5 \\ \hline\end{array}$

Es de destacar el aumento notable en la producción de Méjico (20\%) y Brasil (18\%).

Puestos en forma de gráfico los datos anteriores, obtendríamos el siguiente esquema, en el que se detalla la posición de España, cuyas cifras se han mencionado anteriormente.
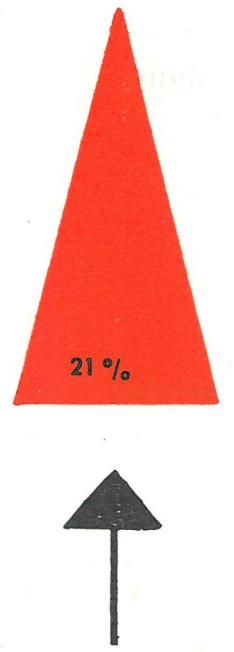

ASIA

$Y$

OCEANIA

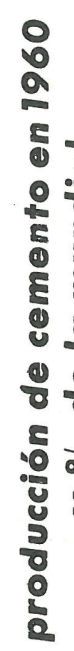

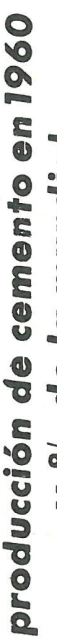

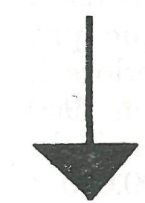

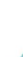

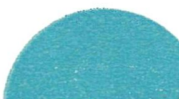

44,4 inillones

$(14 \%)$

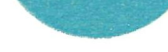

(1) Cembureav. $-93,6=29,6 \%$
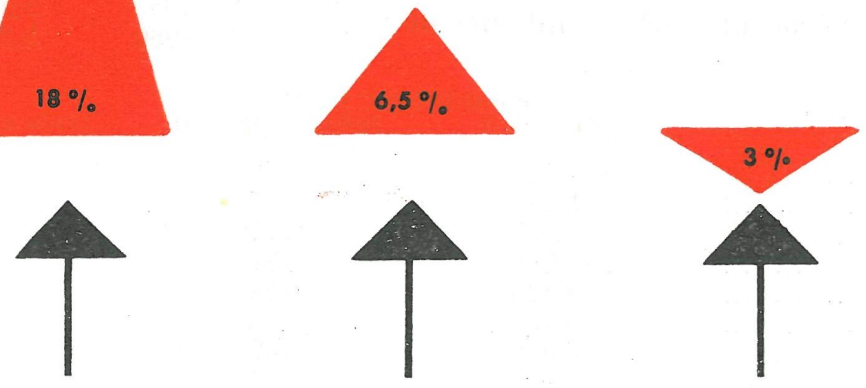

EUROPA

ASIA Y EUROPA
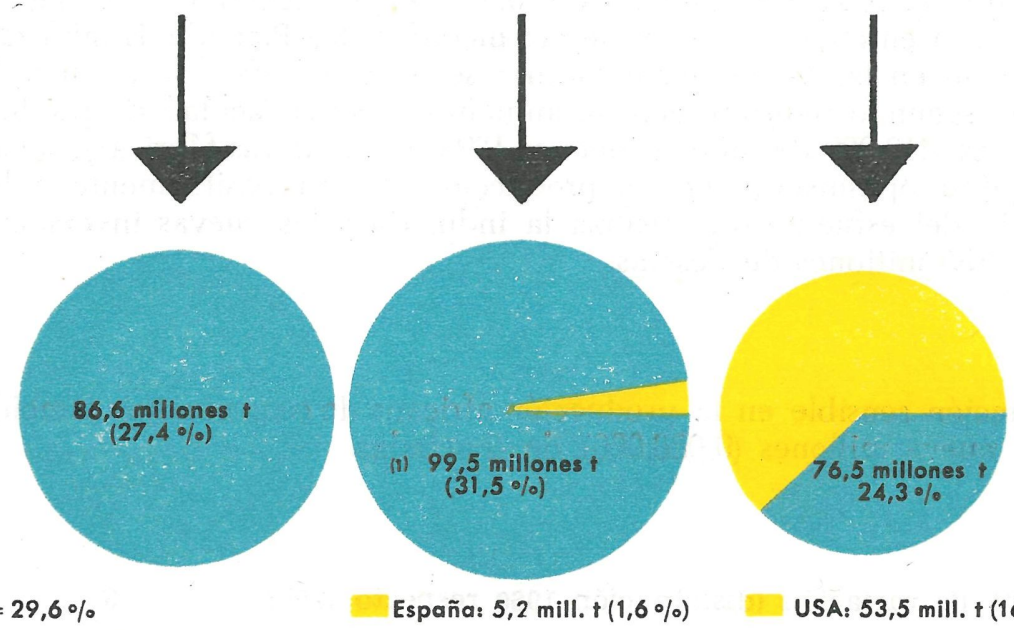

USA: 53,5 mill. $+(16,9 \%)$

\author{
porcontaje de aumento en la \\ producción mundial de cemento \\ (1960 respecto 1959)
}

AMERICA

AFRICA
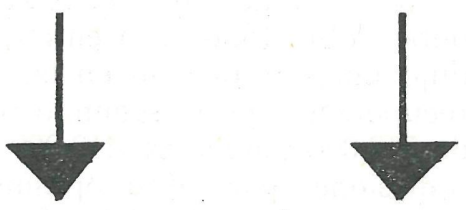

Como puede apreciarse, el período considerado ha sido de gran aumento en la producción mundial de cemento, cuya proyección futura debe considerarse, ya que una expansión en la producción de un $5 \%$ anual la duplica en quince años, lo que puede llevar a una situación de 
superproducción, si no se acompasa con la coyuntura económica general, puesto que existe gran correlación entre ésta y la construcción de viviendas, y estas construcciones con la venta de cemento. Aunque la tasa de crecimiento es muy elevada, parece que las necesidades mundiales y el previsible aumento en el nivel de vida, sobre todo en los países «en plan de desarrollo», aseguran la venta de cemento mundial a pesar del gran aumento en la producción, durante muchos años.

Una tasa de aumento equivalente al del último período considerado, es decir, del 8 al $9 \%$, duplicaría la producción en siete años, lo que debe tenerse en cuenta para evitar desfasajes entre producción y consumo de cemento.

Es de interés citar a este respecto las reuniones celebradas en marzo de 1961, por el Cembureau, para efectuar un «inventario de trabajos de previsión», con análisis, recopilación e intercambio de los métodos usados para ello en los distintos países, con el fin de intentar seleccionar los mejores sistemas de prever el futuro, habida cuenta la coyuntura económica mundial y su posible evolución, necesidades y capacidad para satisfacerlas, con evitación de sobrantes, baja de precios, lucha ruinosa por competencia intensiva y precios dumping, etc., etc.

Esta interesante y necesaria cooperación internacional trata de, en bien de todos, efectuar una previsión de consumo para los diez años próximos y organizar la política de extensión en la capacidad de producción según se prevea la evolución de la economía general, ocupándose también, de forma paralela, en efectuar los mayores esfuerzos posibles para la promoción de ventas y colocación de excedentes en las mejores condiciones posibles y en los países más necesitados, considerando también las condiciones de la competencia ofrecida por los países del bloque oriental.

Entramos así en otra consideración del mayor interés:

\section{El comercio internacional de Cemento}

En el período que comentamos en este trabajo, es decir, en el 1959 y 1960, últimos de los que existen datos, el comercio internacional de cemento ha disminuído en un $2,5 \%$, sin tener en cuenta los intercambios inter-regionales del «bloque comunista», que a estos efectos se considera como un comercio interior entre ellos, siendo de interés destacar también las menores exportaciones realizadas dentro de dicho bloque por algunos países como Rumania, Checoslovaquia y Alemania Oriental.

Las cifras correspondientes son:

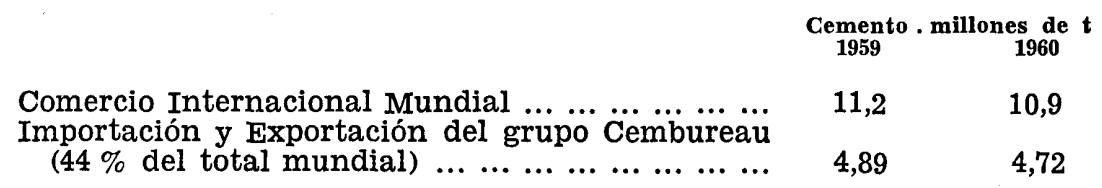

Respecto a las exportaciones de los países europeos, cabe indicar que la mayor parte han sido efectuadas a su zona de influencia, siendo realizadas, principalmente, por Francia y Gran Bretaña, que han embarcado más del $83 \%$ del total, y también Portugal y Turquía.

En cuanto a España, la evolución del comercio exterior de cemento ha presentado en los últimos años el siguiente detalle:

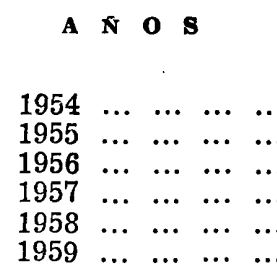

\begin{tabular}{cccr}
\multicolumn{2}{c}{$\begin{array}{c}\text { COMERGIO EXTERIOR } \\
\text { IMPORTAGION }\end{array}$} & DE & \multicolumn{2}{c}{ GEMENTO EN ESPANA } \\
$\mathbf{t}$ & $\$$ & \multicolumn{1}{c}{ EXPORTAGION } \\
147 & 335.830 & 13 & $\$$ \\
49.792 & 1.211 .726 & 3.509 & 18.991 \\
59.637 & 1.853 .819 & 2.826 & 80.815 \\
42.360 & 1.105 .001 & 11.002 & 52.550 \\
10.213 & 278.021 & 1.973 & 192.363 \\
7.219 & 183.913 & 1.215 & 45.476 \\
& & & 45.338
\end{tabular}

Existe, por tanto, una gran anomalía en la exportación española de cemento, con un máximo en 1957 en cuanto a cantidad, y unas cifras prácticamente estabilizadas alrededor de los 45.000 
dólares solamente en los últimos años. En suma, una exportación esporádica y de coyuntura no firme y permanente, frente a una importación de iguales características influída en 1955-56-57, por las compras motivadas para la construcción de Bases americanas.

En cuanto al resto del mundo, Japón experimenta un gran aumento en sus exportaciones, pasando de 1.478 .000 t, en 1959, y 1.617.000, en 1960 (1), y también debe mencionarse Irak, disminuyendo, sin embargo, sus operaciones Yugoslavia, Canadá, India y Ceylán.

En síntesis, cabe decir que el Comercio Internacional del Cemento viene disminuyendo de manera constante, en los últimos años, según el siguiente detalle:

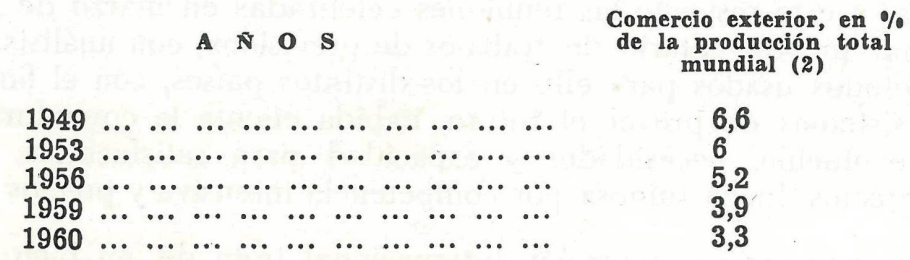

Hay que indicar, además, que los precios internacionales del cemento se han mantenido prácticamente iguales en 1960 respecto a 1959 e, incluso, han disminuído en algunos países como Dinamarca, Finlandia, Gran Bretaña, Países Bajos y Suecia, si bien en reducida proporción que oscila entre $1,4 \%$ y $3,2 \%$.

\section{Conclusión}

Resumiendo, debemos destacar que mientras la producción mundial de cemento aumentó a pesar de las circunstancias internacionales, en 1959-60 (últimos datos disponibles), el comercio internacional del mismo disminuye, en parte, porque algunos países han montado su propia producción, pero también porque no existen demasiados sobrantes y la mayor cantidad producida se consume en el interior como consecuencia del aumento en el nivel de vida y en los nuevos empleos del cemento, no sólo para carreteras, sino también para múltiples usos (traviesas de hormigón, cercos para ventanas, losetas prefabricadas, etc., etc.). En todo caso existe una gran correlación entre el consumo de cemento y el nivel de vida, e incluso, éste es medido por numerosos economistas a través del índice de consumo de cemento.

Es extraordinariamente importante la cooperación internacional para tratar de fijar la coyuntura económica mundial y evitar, en la medida de lo posible, desfasajes excesivos entre producción y consumo. Se hace imprescindible la colaboración para intercambios en la información, promoción conjunta de ventas y los estudios económicos efectuados a través de los organismos centralizadores en los que se analicen los múltiples aspectos de interés común, tales como los nuevos usos del cemento, la competencia de otros materiales de construcción (especialmente los siderúrgicos, aluminio, etc., etc., de uso creciente y también los plásticos, de gran empleo en muros, paneles, etc., etc., la del asfalto para carreteras, etc.), la necesidad de evitar desfasajes entre producción y consumo de cemento, determinación de especificaciones del mismo, control de su empleo, ensayos, calidad, estandarización y normalización, centralización de datos estadísticos e información económica sobre producción, consumo interior, exportación, derechos de aduanas, impuestos, amortizaciones, estructura de su utilización final, cuestiones de mano de obra, salarios, cargas sociales, etc., etc., condiciones de trabajo, examen comparativo de estructuras de precios, forma de transportes, distribución de costes, etc., etc.

He aquí una importante tarea en la que España, con una producción que nos coloca entre los primeros países europeos, ha de colaborar eficazmente dentro de las circunstancias anteriormente descritas, y de nuestra participación en los trabajos correspondientes como miembros de la antigua O. E. C. E., hoy O. C. D. E., del Cembureau, etc., y posiblemente en un futuro próximo de la Comunidad Económica Europea (C. E. E.) o Mercado Común.

(1) Véanse los comentarios a la exportación japonesa de cemento en la Monografía núm. 210 del mismo autor

(2) Excluyendo intercambios entre los países del Bloque Comunista. 